\title{
The importance of potassium citrate and potassium bicarbonate in the treatment of uric acid renal stones
}

\author{
Michele Barbera $^{1}$, Andreas Tsirgiotis ${ }^{1}$, Mauro Barbera ${ }^{2}$, Quintino Paola ${ }^{1}$ \\ ${ }^{1}$ Operative Unit of Urology, Ospedale Giovanni Paolo II, Sciacca, Italy; \\ ${ }^{2}$ University of Palermo, Palermo, Italy.
}

\begin{abstract}
Summary Uric acid calculi can also be treated without surgery, with simple medical lytic therapy. After appropriate dietary adjustments and add of mineral water, the needed amount of alkali supplementation can increase $\mathrm{pH}$ values of the urine in order to dissolve the stones. Treatment should be prolonged to prevent stone recurrence. A case of bilateral renal uric acid stones that were successfully treated by alakalizing treatment was presented.
\end{abstract}

KEY WORDS: Potassium citrate; Uric acid stones.

Submitted 15 November 2016; Accepted 15 December 2016

\section{INTRODUCTION}

The changed dietary habits, related to feeds oriented to excessive intakes of protein or fat, tend to move the body's metabolic balance towards excessive acid load (1). This condition would facilitate, if persisting, the onset of certain diseases, such as osteoporosis and renal calculi (2), which often occur, just in the presence of reduced citrates in the urine and of a urinary acidic $\mathrm{pH}$ (5.0-5.5). The maintenance of hyperacidic values of urinary $\mathrm{pH}$ more easily induces the precipitation of uric acid in the urine and the consequent formation of uric acid stones.

Uric acid stones account for about 10\% of all urinary stones. The incidence of uric lithiasis increases significantly with age. Uric acid is very slightly soluble in an acidic $\mathrm{pH}$, so that the formation of uric acid crystals is practically mandatory when the $\mathrm{pH}$ of the urine is persistently below 5.5.

Predisposing causes:

- diabetes mellitus (insulin resistance that characterizes both the metabolic syndrome that type 2 diabetes decreases the urinary $\mathrm{pH}$ ) (1);

- obesity (those with a high body mass index (BMI) have higher urinary excretion of oxalate, urate, sodium and phosphate) BMI has an inverse correlationwith the $\mathrm{pH}$ and uric acid urinary supersaturation (not calcium oxalate) is directly related to BMI;

- hyperuricosuria and hyperuricemia.

\section{CAse Report}

A 59 year old male patient with diabetes mellitus and heart disease (who underwent coronary heart bypass

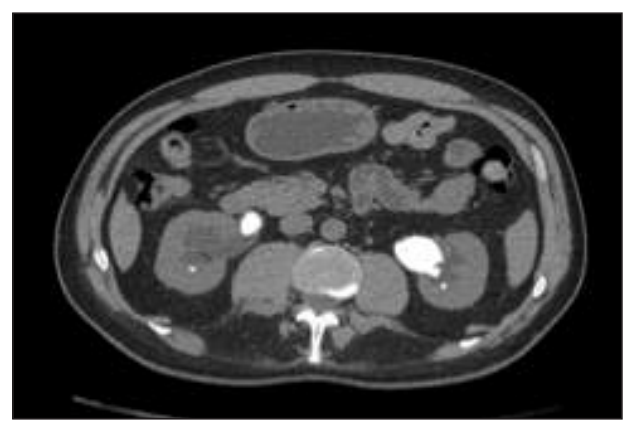

Figure 1.

CT pre

treatment

surgery 6 months before and was receiving long-term anticoagulant treatment) was affected by bilateral renal calculi.

Abdominal computed tomography (CT) without contrast confirmed the presence of bilateral pelvic renal stones measuring $30 \times 20 \mathrm{~mm}$ in the right kidney and 40 x $30 \mathrm{~mm}$ in the left kidney (Figure 1).

Renal stone density was measured according the Hounsfield Scale (3) that defines as zero Hounsfield Unit (HU) the radiodensity of water and around $1000 \mathrm{HU}$ the radiodensity of bones.

The density of the patient's stones ranged between 400 and $500 \mathrm{HU}$, is a typical value of uric acid stones. The patient underwent a therapy with a mixture of potassium citrate (2) and potassium bicarbonate (LITHOSOLV) with the aim to alkalize the urine to achieve $\mathrm{pH}$ values between 6.5 and 6.8. The $\mathrm{pH}$ of the urines was periodically checked with litmus test and colorimetric comparison table, in order to daily adjust the dose.

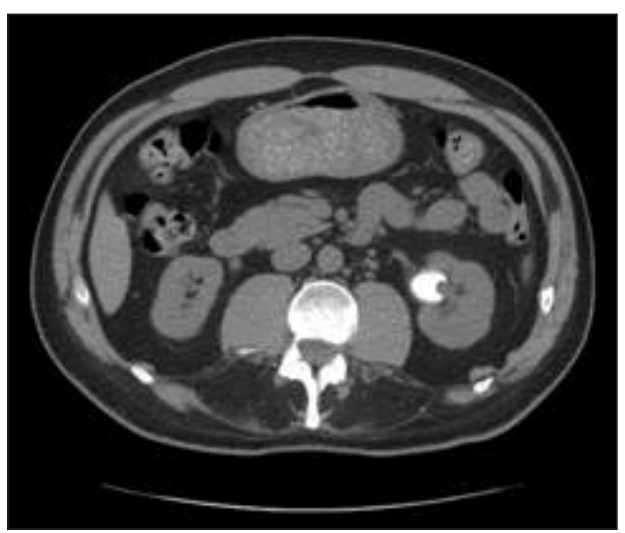

Figure 2.

CT after

4 months. 


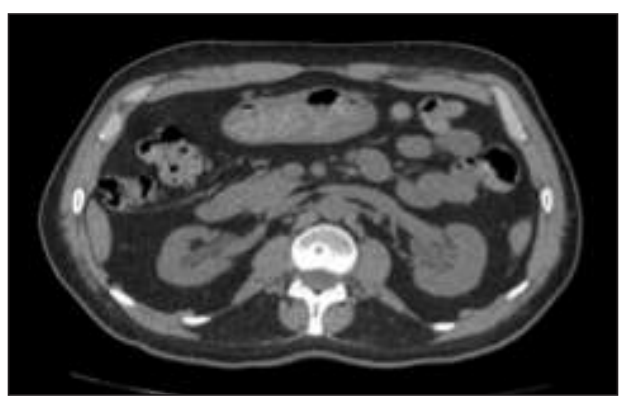

Figure 3.

CT after

7 months.

After 4 months of therapy an abdomen CT without contrast highlighted the disappearance of the stones in the right renal pelvis and the significant reduction of the stones in the left kidney (Figure 2).

After 7 months of treatment an abdomen CT without contrast was repeated to show total disappearance of the stone in the left kidney (Figure 3).

\section{Discussion}

The primary goal of the medical treatment of uric acid stones is the reduction of urinary supersaturation for uric acid increasing urinary volume and urinary $\mathrm{pH}$ (to 6.57) rather than reducing urinary uric acid excretion.

The modification of acidic urinary $\mathrm{pH}$ with alkalizing agents would favor the solubilization of urinary uric acid and the dissolution of the stone.

A diet rich in fruits and vegetables is the main source of alkalizing substances (3). However, a dietary only approach is not always sufficient, and food supplements may be needed.

This occurs mainly in the elderly, in people performing intense physical activity, in postmenopausal women or in those with highly protein diets.
The treatment should include:

- drinking mineral water in order to achieve an urine output of about 2 liters per day;

- a diet limiting foods of animal origin (meat, fish, offal, etc.) and mostly vegetarian should be suggested in order to decrease the load of fixed acids and purines;

- food supplementation by potassium alkali salts in order to correct the excessive acid load.

The $\mathrm{pH}$ measurement can be carried out easily through the use of diagnostic strips.

The urinary $\mathrm{pH}$ value varies along the day with the most significant drop in the course of the night and in the first morning. For thisreason it is preferable to measure it after awaking.If the $\mathrm{Ph}$ is below 6 , it should be adjusted by taking the alkali supplement in the morning and evening. Each dose provides a quantity of potassium in the form of citrate and potassium bicarbonate equal to $1.173 \mathrm{~g}$ (30 $\mathrm{mEq}$ ). The total dose may be increased until to 3 administrations a day. The effect of alkalizing action of urine could be verified again by using diagnostic strips.

Use of allopurinol (150-300 mg, once a day) could be appropriate in presence of hyperuricemia and/or excessive urinary uric acid.

\section{REFERENCES}

1. Abate N, Chandalia M, Cabo-Chan AV Jr, et al. The metabolic syndrome and uric acid nephrolithiasis: novel features of renal manifestation of insulin resistance. Kidney Int. 2004; 65:386-392.

2. Pak CYC, Sakhaee K, Fuller C. Successful management of uric acid nephrolithiasis with potassium citrate. Kidney Int. 1986; 30:422-428.

3. Welk BK, Teichman JM Uric acid nephrolithias in the era of noncontrast computed tomography, CUAJ. 2008; 2:420-421.

\section{Correspondence}

Michele Barbera, MD

barbera.mic@gmail.com

Andreas Tsirgiotis, MD

atsirgiotis@alice.it

Paola Quintino, MD

q.paola@inwind.it

Operative Unit of Urology, Ospedale Giovanni Paolo II, Sciacca, Italy

Mauro Barbera, MB

barbera.mau@gmail.com

University of Palermo, Palermo, Italy 